\title{
Dispersive Fourier transform using few-mode fibers for real-time and high-speed spectroscopy
}

\author{
Yi Qiu, Chi Zhang, Kenneth K. Y. Wong, Kevin K. Tsia* \\ Department of Electrical and Electronic Engineering, The University of Hong Kong, Pokfulam Road, \\ Hong Kong. \\ *Email: tsia@hku.hk
}

\begin{abstract}
Dispersive Fourier Transform (DFT) is a powerful technique for real-time and high-speed spectroscopy. In DFT, the spectral information of an optical pulse is mapped into time using group velocity dispersion (GVD) in the dispersive fibers with an ultrafast real-time spectral acquisition rate (>10 MHz). Typically, multi-mode fiber (MMF) is not recommended for performing DFT because the modal dispersion, which occurs simultaneously with GVD, introduces the ambiguity in the wavelength-to-time mapping during DFT. Nevertheless, we here demonstrate that a clear wavelength-to-time mapping in DFT can be achieved by using the few-mode fibers (FMFs) which, instead of having hundreds of propagation modes, support only a few modes. FMF-based DFT becomes appealing when it operates at the shorter wavelengths e.g. 1- $\mu \mathrm{m}$ range--a favorable spectral window for biomedical diagnostics, where low-cost single mode fibers (SMFs) and high-performance dispersion-engineered fibers are not readily available for DFT. By employing the telecommunication SMFs (e.g. SMF28), which are in effect FMFs in the 1- $\mu \mathrm{m}$ range as their cut-off wavelength is $\sim 1260 \mathrm{~nm}$, we observe that a $3 \mathrm{~nm}$ wide spectrum can be clearly mapped into time with a GVD as high as $-72 \mathrm{ps} / \mathrm{nm}$ and a loss of $5 \mathrm{~dB} / \mathrm{km}$ at a spectral acquisition rate of $20 \mathrm{MHz}$. Moreover, its larger core size than the high-cost 1- $\mu \mathrm{m}$ SMFs renders FMFs to exhibit less nonlinearity, especially high-power amplification is implemented during DFT to enhance the detection sensitivity without compromising the speed. Hence, FMF-based DFT represents a cost-effective approach to realize high-speed DFT-based spectroscopy particularly in the biomedical diagnostics spectral window.
\end{abstract}

Key words: spectroscopy, dispersive Fourier transform, group velocity dispersion, dispersive fibers, ultrafast real-time spectroscopy, few-mode fibers, biomedical diagnostics

\section{INTRODUCTION}

Optical spectroscopy has been well-recognized as an excellent toolset which unravels multitude of information related to the material properties. In the realms of basic life science research and clinical diagnostics, high-speed and real-time spectroscopy is particularly important for improving both the measurement efficacy and efficiency by providing fast dynamical information, or delivering high-throughput and high content screening. Since the conventional spectrometers generally adopt mechanical moving parts e.g., a rotating grating or a detector array e.g., a charge-coupled device (CCD) with a limited frame rate typically less than $\mathrm{kHz}$. Moreover, the fundamental tradeoff between sensitivity and speed, i.e. higher acquisition speed can be achieved at the expense of the number of collected photon - losing the detection sensitivity. Therefore, traditional spectrometers are not favorable for ultrafast real-time measurements. To this end, wavelength-time spectroscopy, which is also known as dispersive Fourier transform (DFT) spectroscopy [1-3], has been demonstrated to perform ultrafast real-time spectroscopy with spectral acquisition rate of $>\mathrm{MHz}$, a speed that cannot be achieved by conventional spectrometers. It can also be applied to ultrafast optical imaging [4-7]. In DFT, the spectral information of an optical broadband pulse is mapped into time using group velocity dispersion. A single photo-detector and a high-speed electronic digitizer are used to capture the spectra in real time. By far, the common operation wavelength range for DFT is the telecommunication wavelength band around $1500 \mathrm{~nm}$ due to the fact that highly-

Optical Fibers and Sensors for Medical Diagnostics and Treatment Applications XII, edited by Israel Gannot, Proc. of SPIE Vol. 8218, 82180P - @ 2012 SPIE

CCC code: $1605-7422 / 12 / \$ 18 \cdot$ doi: $10.1117 / 12.907785$ 
dispersive fibers (e.g. dispersion compensation fiber) are widely accessible for long-haul telecommunication. Since the favorable spectral window for biomedical diagnostics is at $\sim 1 \mu \mathrm{m}[8$, operating DFT at $\sim 1 \mu \mathrm{m}$ can open up a new avenue for ultrafast real-time biomedical measurements. However, enabling DFT in the $1-\mu \mathrm{m}$ regime is not a trivial task because of the fact that low-cost single-mode fibers (SMFs) and high dispersion fibers are not readily available in this wavelength range. In this paper, instead of using the specialty single-mode fiber at $\sim 1 \mu \mathrm{m}$, we demonstrate a cost-effective alternatives of operating DFT at $\sim 1 \mu \mathrm{m}$ using the standard telecommunication fibers (e.g. SMF28, dispersion compensation fiber (DCF))which in effect are the few-mode fibers (FMFs) at this wavelength range. Since the cutoff wavelengths of these telecommunication fibers are typically $>1 \mu \mathrm{m}$, (e.g. for SMF28, the cutoff wavelength is $\sim 1200 \mathrm{~nm}$ ), only a few modes, instead of hundreds of modes, can thus be supported by the FMFs $[9,10]$.

Multi-mode fibers are generally not recommended to perform DFT due to the fact that different GVD of different modes will lead to the ambiguity in the wavelength-time mapping. However, we experimentally demonstrated that under proper input coupling conditions the fundamental mode can be primarily excited and propagates along the FMF (both SMF28 and DCF). In our experiments, we observed that relatively high dispersion, which is sufficient for practical DFT spectroscopy, is attained in the FMFs. We also evaluated and compared the dispersion-to-loss ratios among the three types of fibers, i.e. the 1- $\mu \mathrm{m}$ SMF, and the two FMFs: SMF28 and DCF. We note that the tradeoff between dispersion and optical loss can be mitigated by means of simultaneous optical amplification such as Raman amplification within the fibers during the DFT process known as amplified DFT (ADFT).[1-3]

\section{WORKING PRINCIPLE}

In a DFT process, the spectral information is mapped into the time domain using GVD. Dispersive fiber and a single photo-detector is used in the DFT system instead of spatial disperser (i.e. diffraction grating) and detector array (e.g. CCD) which greatly simplify the system and help realizing ultra-fast and real-time spectroscopic measurement by allowing the optical spectrum to be mapped in time domain directly. The spectral acquisition rate is hence determined by the repetition rate of the broadband laser source, which is typically $>\mathrm{MHz}$ - orders-of-magnitude faster than the conventional spectrometers. Since the loss always comes with dispersion, there is a tradeoff between large loss and high dispersion. However, this tradeoff can be compromised by simultaneously amplifying the signal in the optical domain which is known as ADFT.

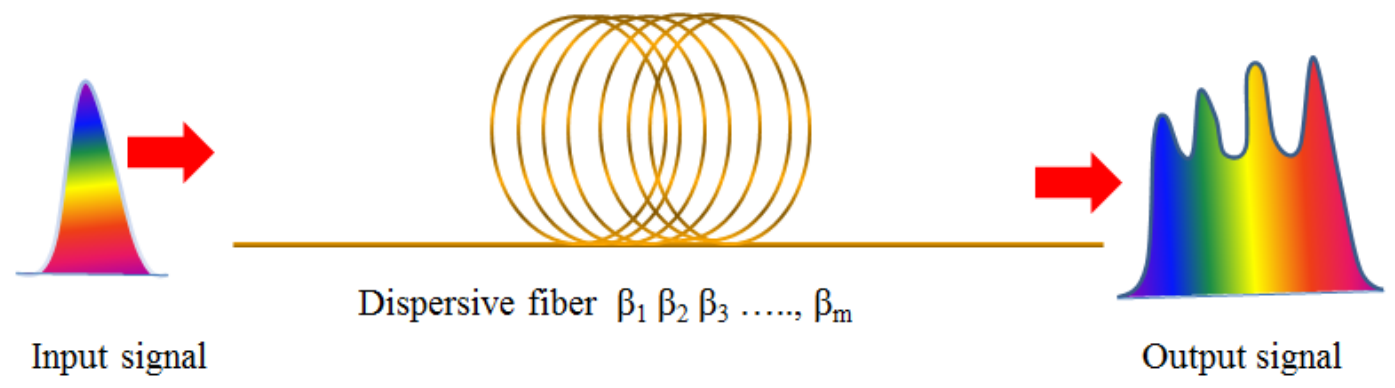

Figure 1 Working principle for dispersive Fourier transform (DFT) in which the spectral information is mapped into the time domain. $\beta_{\mathrm{m}}$ are the GVD coefficients of the dispersive fiber. 


\section{EXPERIMENTAL SET UP}

We employed a mode-locked laser at the center wavelength of $1064 \mathrm{~nm}$ with a repetition rate of $20 \mathrm{MHz}$ to pump a $20-\mathrm{m}$ highly nonlinear photonics crystal fiber (PCF), with a zero-dispersion wavelength of $\sim 1060 \mathrm{~nm}$, to generate a supercontinuum (SC) spanning from $\sim 800 \mathrm{~nm}$ to $1300 \mathrm{~nm}$. A weak continuous-wave (CW) laser, at a wavelength near the modulation instability (MI) gain spectral peak in the SC, was launched together with the pump into the PCF. This CW laser, combined with the pump by a beam splitter (BS) with the splitting ratio of 98:2, acts as a minute trigger which can enhance the SC power and greatly improves the pulse-to-pulse temporal stability of the SC [11,12]. A 4-f system was introduced with two diffraction gratings (grove density 800 lines $/ \mathrm{mm}$ ) and a pair of lenses (focal length $f=75 \mathrm{~mm}$ ) and a spatial mask, which is used to generate the arbitrary spectral shapes. The shaped spectrum will then be mapped into the time domain by the DFT process in the 1- $\mu \mathrm{m}$ SMF, SMF28 and DCF. We captured and compared the DFT waveforms using the 1- $\mu \mathrm{m}$ SMF, SMF28 and DCF by a real time oscilloscope (16GHz, 40GS/s) (Fig.2). We found that the fundamental mode can primarily be excited in the FMFs by connecting a SMF patch cord to the FMF through the standard telecommunication fiber connector. This simple configuration would minimize any lateral misalignment between the two fibers and ensure that only the fundamental mode can be allowed. The single-mode guiding was verified by capturing the mode-profiles at the FMF outputs using a near-infrared camera.

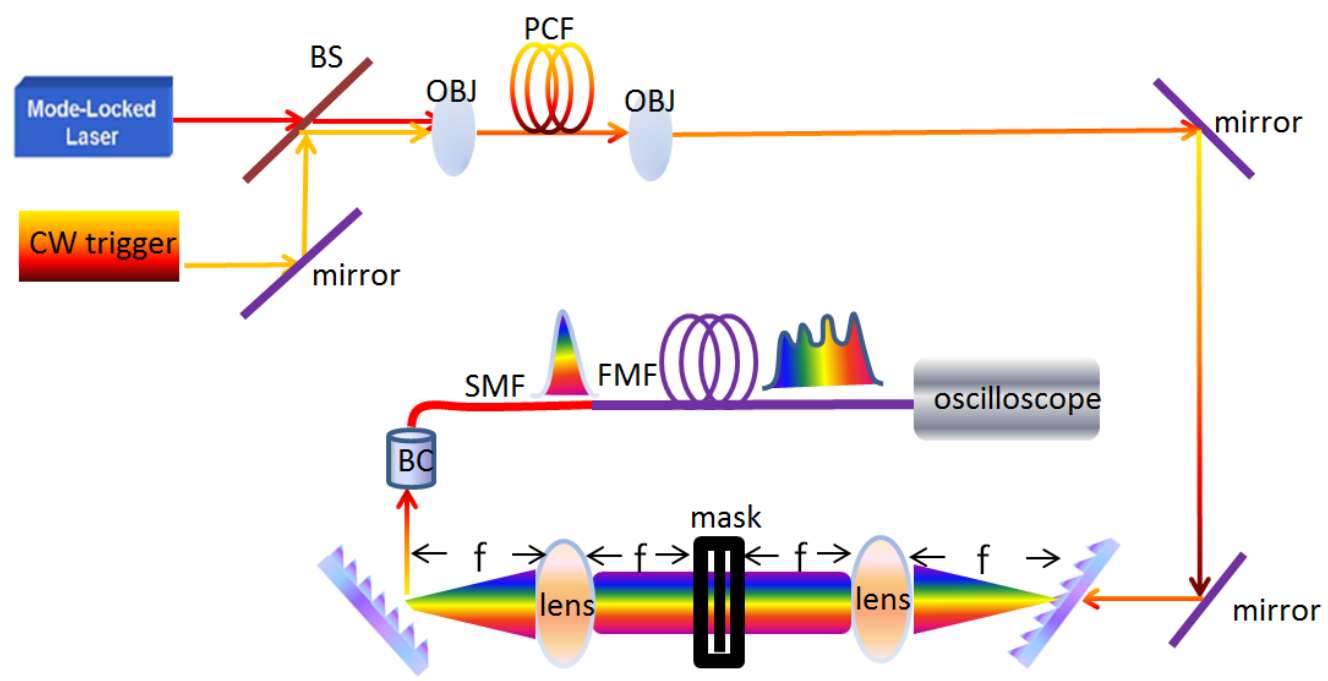

Figure 2. Experimental set up for dispersive Fourier transform (DFT) using FMF.

\section{RESULTS AND DISCUSSION}

Figure 3 shows the basic performance of the DFT at 1- $\mu \mathrm{m}$ using a $5-\mathrm{km}$-long $1 \mu \mathrm{m}$ SMF. A spectral acquisition rate as high as $5 \mathrm{MHz}$ can be clearly evident in the captured waveform. Note that each pulse corresponds to a single-shot 
wavelength-time-mapping by means of GVD. In this wavelength-time mapping, the 2-peak feature is clearly mapped into the time domain with a reasonably good pulse-to-pulse instability which is enabled by the CW-triggering mechanism [11,12]. Based on the temporal width and the spectral bandwidth defined by the grating-pair shaper (shown in Fig. 2), we estimated a total dispersion of the $1 \mu \mathrm{m}$ fiber to be $\sim-150 \mathrm{ps} / \mathrm{nm}$.
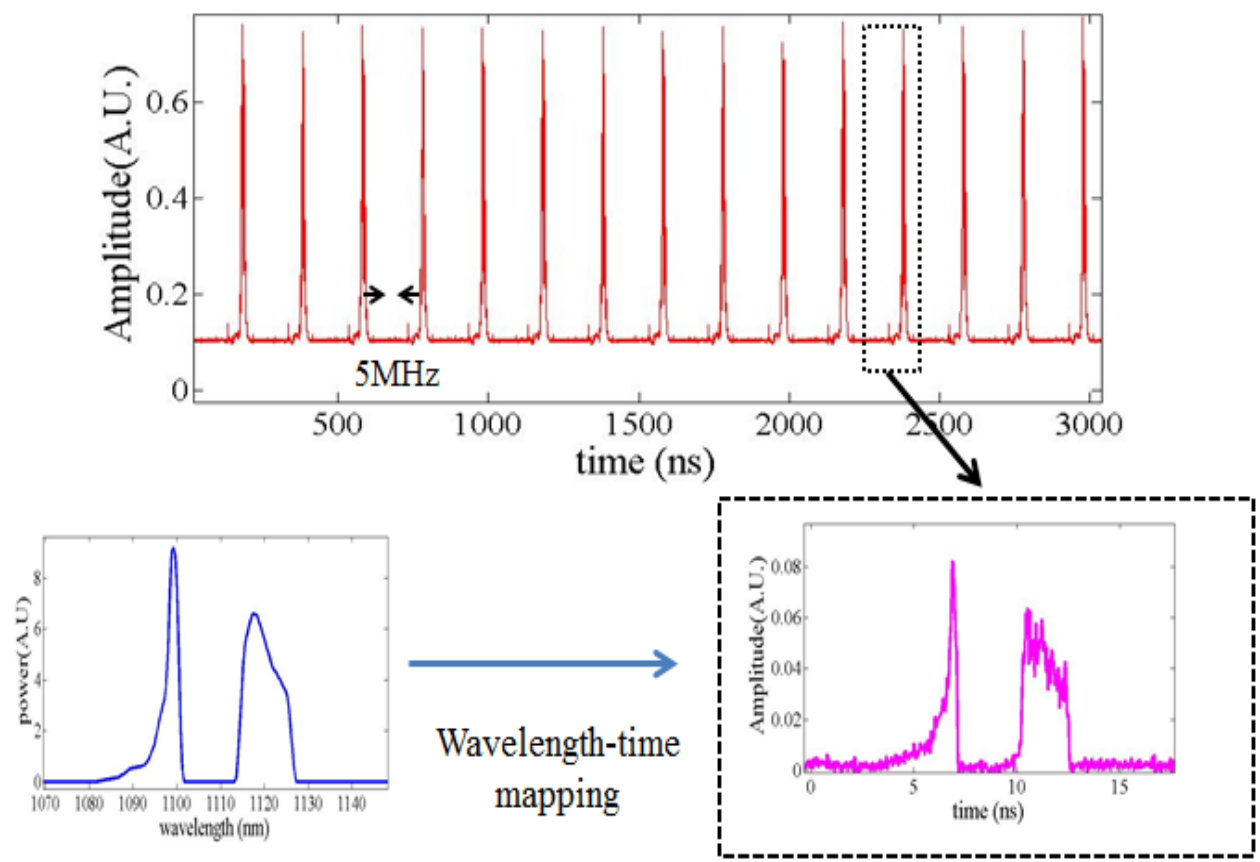

Fig 3. The spectral information (blue) of an optical pulse is mapped into time (magenta) using group velocity dispersion (GVD) with a frame rate of $5 \mathrm{MHz}$

Figure 4 shows the comparison of the DFT operations between the different types of fibers (SMF28 (Fig. 4(b)), 1 $1 \mu \mathrm{m}$ fiber (Fig. 4(c)), and DCF (Fig. 4(d))). The mode profile of each fiber is imaged by the infrared camera at the end facet of each fiber. In general, the spectral information can be clearly mapped into the time domain in all three fibers indicating that primarily the fundamental mode is attributed to the DFT process in the FMF and no higher-order modes (thus the mode coupling) occur which could create the ambiguity of the wavelength-to-time mapping except in the case of DCF. Based on the captured images of the mode-profiles, both 1- $\mu \mathrm{m}$ SMF and the SMF28 show the clear single-mode profiles whereas the mode pattern of the DCF shows some minimal distortions. This could be due to the mode coupling between the fundamental mode and the higher-order modes. Further investigation will be carried out to quantify the mode-coupling effect in the FMFs. Nevertheless, we show that the current coupling configuration, i.e. using a standard fiber connector, is a feasible and handy approach to ensure single-mode guiding in the FMFs - one key requirement for performing good-quality DFT, albeit the additional coupling loss due to the core-size mismatch between the SMF ( 2$4 \mu \mathrm{m})$ and FMF $(5-8 \mu \mathrm{m})$.

Based on the temporal widths of the captured waveforms, we estimated the GVDs of the fundamental modes in SMF28 and DCF are $-3 \mathrm{ps} / \mathrm{nm} \cdot \mathrm{km}$ and $-150 \mathrm{ps} / \mathrm{nm} \cdot \mathrm{km}$ respectively near the $1 \mu \mathrm{m}$ range, both of which lie in the anomalous region. A 10-nm wide spectral feature can be clearly resolved in the time domain for both SMF28 and DCF. Fig. 5 shows another DFT operation of the SMF28 that can resolve even narrower spectral feature (3-nm in width). Since GVD is 
generally wavelength dependent, a more accurate measurement of the dispersion curve near $1 \mu \mathrm{m}$ can be performed by a technique based on optical low coherence reflectometry [13]. In the DFT process, the loss of SMF28 and DCF are $5 \mathrm{~dB} / \mathrm{km}$ and $76 \mathrm{~dB} / \mathrm{km}$, respectively. The dispersive loss can be compensated by simultaneous optical amplification within the fibers. High-gain and wideband optical amplification in the $1-\mu \mathrm{m}$ range can be achieved by fiber Raman amplification, fiber optical parametric amplification [3, 4]. Off-the-shelf Ytterbium-doped fiber amplifiers (YDFAs) and $1-\mu \mathrm{m}$ semiconductor optical amplifiers (SOAs) can also be utilized to serve as the additional amplification mechanisms to enhance the detection sensitivity of the FMF-based ADFT[14]. We note that high-order modes can be selectively excited in the FMF fiber by the fiber fusion-splicing technique, e.g., introducing an offset between the SMF and FMF at the connected facet. And these high-order modes can provide significantly higher dispersion compared with the fundamental mode which can potentially be exploited as another way to perform DFT, but with a much shorter fiber length.

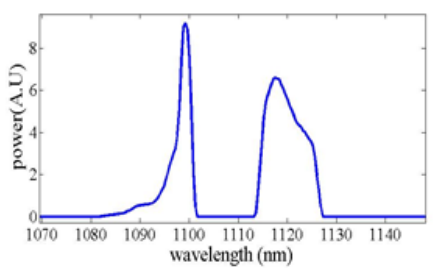

(a)

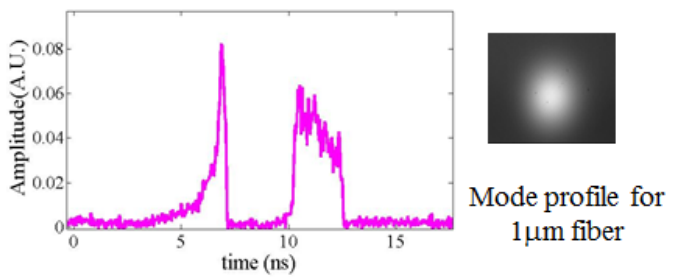

(c)

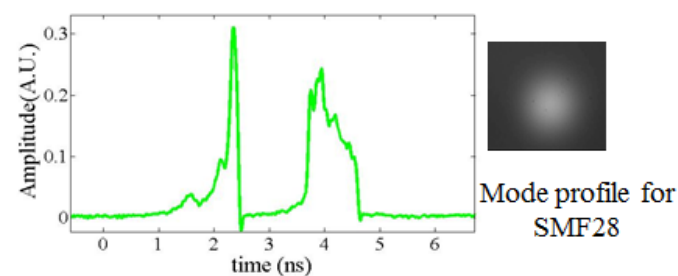

(b)

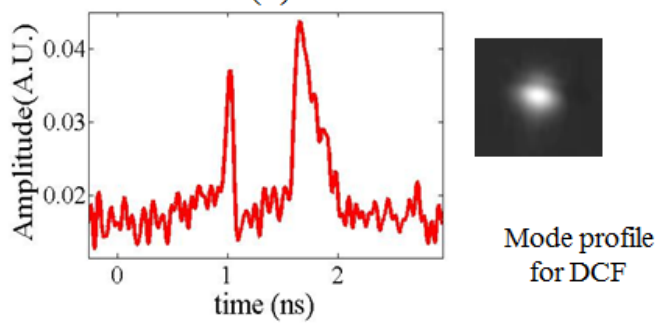

(d)

Fig 4 Wavelength-time mapping of (b) $24-\mathrm{km}$ SMF 28 with core diameter of $8 \mu \mathrm{m}$ and,(c) 5 -km $1 \mu \mathrm{m}$ fiber with core diameter of $4 \mu \mathrm{m}$ and (d)a 200-m DCF with core diameter of $5 \mu \mathrm{m}$. The original spectrum is shown in (a). Insets: output mode profiles of the fibers.
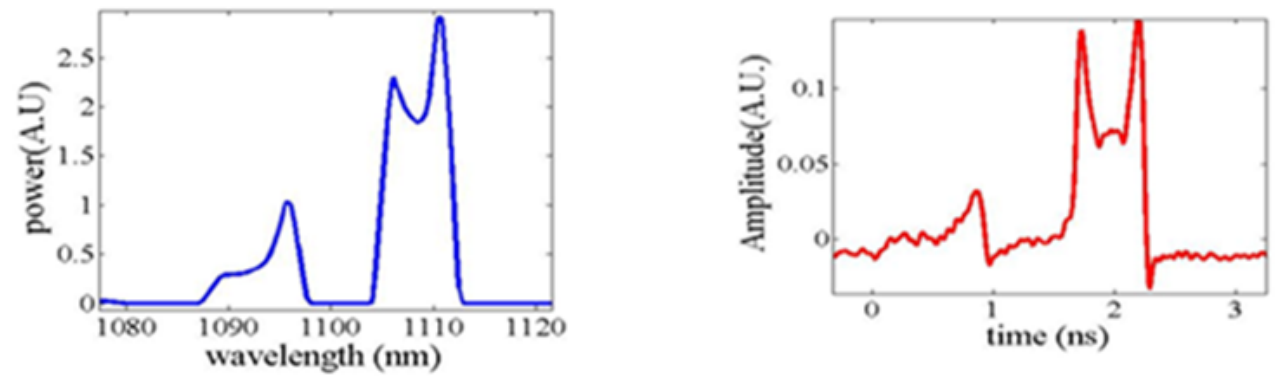

Fig 5. Wavelength-time mapping of SMF28 in which a 3nm wide spectral feature can be clearly mapped into the time domain 


\section{CONCLUSION}

In summary, a cost-effective approach for realizing DFT-based spectroscopy at the 1- $\mu \mathrm{m}$ range by using standard telecommunication SMF28 and DCF as FMFs instead of the high-cost specialty 1- $\mu \mathrm{m}$ SMF was reported. Using a straight-forward coupling approach, only the fundamental mode can primarily be excited and propagates in the FMFs. The dispersion of the SMF28 and DCF can be as high as to $-3 \mathrm{ps} / \mathrm{nm} \cdot \mathrm{km}$ and $-150 \mathrm{ps} / \mathrm{nm} \cdot \mathrm{km}$ with the loss $5 \mathrm{~dB} / \mathrm{km}$ and $76 \mathrm{~dB} / \mathrm{km}$, respectively. While the loss of DCF would be too high for practical applications, SMF28 shows a reasonable GVD and acceptable loss level (comparable to the 1- $\mu \mathrm{m} \mathrm{SMF).} \mathrm{Hence,} \mathrm{together} \mathrm{with} \mathrm{implementing} \mathrm{the} \mathrm{simultaneous}$ optical amplification, SMF28 could be a viable candidate for performing FMF-based ADFT at the 1- $\mu$ m wavelength regime. It represents a cost-effective approach to realize high-speed ADFT-based spectroscopy particularly in the biomedical diagnostics spectral window.

\section{ACKNOWLEDGEMENT}

This work is partially supported by grants from the Research Grants Council of the Hong Kong Special Administrative Region, China (project nos. HKU 7179/08E, HKU 7183/09E, and HKU 717510E).

\section{REFERENCE}

[1]D. R. Solli, J. Chou, and B. Jalali, "Amplified wavelength-time transformation for real-time spectroscopy," Nature Photonics, 2, 48 (2008).

[2] J. Chou, D. R. Solli, and B. Jalali, " Real-time spectroscopy with subgigahertz resolution using amplified dispersive Fourier transformation" Appl. Phys. Lett. 92, 111102 (2008).

[3] K. Goda, D. Solli, K. K. Tsia, and B. Jalali, "Theory of amplified dispersive Fourier transformation", Phys. Rev. A 80, 043821 (2009).

[4]K. Goda, K. K. Tsia, and B. Jalali, "Amplified dispersive Fourier-transform imaging for ultrafast displacement sensing and barcode reading," Appl. Phys. Lett. 93, 131109 (2008).

[5] K. Goda, K. K. Tsia, and B. Jalali, "Serial time-encoded amplified imaging for real-time observation of fast dynamic phenomena," Nature 458, 1145 (2009).

[6] K. K. Tsia, K. Goda, D. Capewell, and B. Jalali, "Performance of serial time-encoded amplified microscope," Opt. Express 18, 10016 (2010).

[7] C. Zhang, Y. Qiu, R. Zhu, and Kenneth K. Y. Wong, and K. K. Tsia, "Serial time-encoded amplified microscopy (STEAM) based on a stabilized picosecond supercontinuum source," Opt. Express 19, 15810-15816 (2011)

[8] P. N. Prasad, Introduction to Biophotonics, Wiley-Interscience (2003).

[9] F. Yaman, N. Bai, B.Zhu, T.Wang, and G. Li , "Long distance transmission in few-mode fibers" Optics Express, Vol. 18, Issue 12, pp. 13250-13257 (2010)

[10] T.-J. Ahn, Y. Jung, K. Oh , and D. Y. Kim , "Chromatic Dispersion Measurement for a Few-Mode Fiber Using Optical Frequency-Domain Reflectometer, Quantum Electronics and Laser Science Conference (QELS)May 21, 2006

[11] K. K. Y. Cheung, C. Zhang, Y. Zhou, K. K. Y. Wong, and K. K. Tsia, "Manipulating supercontinuum generation by minute continuous wave," Opt. Lett. 36, 160-162 (2011)

[12] Q. Li, F. Li, K. K. Y. Wong, A. P. T. Lau, K. K. Tsia, and P. K. A. Wai, "Investigating the influence of a weak continuous-wave-trigger on picosecond supercontinuum generation," Opt. Express 19, 13757-13769 (2011)

[13] S. Ring, D. Menashe, U. Levy, S. Steinblatt, and Y. Danziger, and Moshe Tur , "Characterization of Mode Coupling in Few-Mode Fibers Using Optical Low-Coherence Reflectometry",OFC/NFOEC (2008)

[14] J. Fan, A. Dogariu, and L. J. Wang, "Parametric amplification in a microstructure fiber," Appl. Phys. B 81, 801-805 (2005). 